\title{
Characterisation of plastic zones around crack-tips in pure single-crystal tungsten using electron backscatter diffraction
}

\author{
J D Murphy, A J Wilkinson and S G Roberts \\ Department of Materials, University of Oxford, Parks Road, Oxford, OX1 3PH, UK \\ E-mail: john.murphy@materials.ox.ac.uk
}

\begin{abstract}
Plastic zones around crack-tips in tungsten were characterised by electron backscatter diffraction (EBSD). Pre-cracks were made in $\sim 1 \mathrm{~mm}$ square cross section beams of pure single-crystal tungsten using a spark erosion method. The beams were loaded at different temperatures to a range of stress intensity factors below the fracture toughness. High resolution EBSD patterns were recorded in a two dimensional array in the vicinity of the crack-tip on sectioned samples. Cross-correlation based analysis of these patterns was used to determine lattice rotations associated with the plastic deformation near the cracks. Crystal rotations in the plane of observation were found to dominate over rotations in other planes. For all specimens deformed above the brittle-to-ductile transition temperature, plastic zones were found to extend from the crack tips on along narrow bands along $\{110\}$ planes. The sizes of the plastic zones and their associated lattice rotations were determined as a function of loading history.
\end{abstract}

\section{Introduction}

Tungsten has been suggested as a plasma-facing material for critical components in fusion reactors, such as ITER, and in such an application it will be subjected to continually high levels of radiation damage, high temperatures and high stresses [1]. Transient events, such as the high strain rates caused by thermal shock due to a local plasma variation, could lead to cracking of the material. It is therefore important to understand the mechanical properties of tungsten, including the role of dislocations around cracks subjected to stress.

At low temperatures, many materials fail by cleavage and exhibit entirely brittle behaviour. At high temperatures, such materials can show ductile behaviour, characterized by significant plastic deformation. A brittle-to-ductile transition (BDT) temperature $\left(T_{B D T}\right)$ can be measured for a particular testing configuration. For many materials, $T_{B D T}$ is found to vary with strain rate according to an Arrhenius relation, with characteristic activation energy, $E_{B D T}$ :

$$
\frac{d \varepsilon}{d t}=A \exp \left(-\frac{E_{B D T}}{k T_{B D T}}\right)
$$

In a study of the BDT in single-crystal tungsten, Gumbsch et al. found $E_{B D T}$ to be approximately $0.2 \mathrm{eV}[2]$. As is the case for most BCC metals, dislocation velocity data for tungsten are scarce and data are only known to be available at $77 \mathrm{~K}$ and room temperature [3]. However, numerical simulations suggest the motion of the screw dislocations that control the rate of plastic deformation [4] is controlled by an activation energy in excess of $1 \mathrm{eV}$ [5]. The low value of $E_{B D T}$ found by Gumbsch et 
al. can be attributed to the crystallography of the particular specimens used for their experiments in which, uniquely, the controlling process is likely to be the glide of edge dislocations. More recent work by Giannattasio and Roberts has found $E_{B D T}$ to be approximately $1.0 \mathrm{eV}$ in both pure polycrystalline and pure single-crystal tungsten [6] and $1.45 \mathrm{eV}$ in less pure sintered material [7]. This implies that, for the general case, the BDT in tungsten is controlled by screw dislocation motion.

In Giannattasio and Roberts' work [6,7], pre-cracked rectangular beams were loaded at a range of strain rates and temperatures. Their measurements were made in terms of a stress intensity factor, $K$, defined by:

$$
K=\sigma \sqrt{\pi c}
$$

where $\sigma$ is the applied stress and $c$ is the depth of the deepest crack measured from the top surface of the specimen. For a specimen which fractures under a stress of $\sigma_{\text {fracture }}$, the stress intensity factor is referred to as the fracture toughness $\left(K_{l c}\right)$. For all strain rates investigated, the value of $K_{l c}$ was found to rise significantly as the temperature approached $T_{B D T}$. This has been modelled as being due to the increasing activity of dislocations in the plastic zone around the crack tip [8,9]; the "shielding" effect of the dislocations reduces the crack-tip stress intensity and thus forces higher external loading to be required for fracture.

In the present study similar specimens were produced to those in Giannattasio and Roberts' work $[6,7]$. The specimens made were loaded at a range of temperatures to different values of $K$ below $K_{I c}$, so that the specimens did not fracture but so that plastic zones would be expected to develop around the cracks. Cross-correlation based analysis of electron backscatter diffraction (EBSD) patterns was then used to map rotations of the lattice in the vicinity of the crack-tip. The results obtained allow the geometry and size of plastic zones around crack-tip to be determined.

\section{Experimental method}

2.1 Specimen production

A single-crystal of pure tungsten was supplied by Metal Crystals and Oxides Limited (Cambridge, UK). This was cut into rectangular beams with faces along $<100>$ directions. These beams measured approximately $1 \mathrm{~mm} \times 1 \mathrm{~mm} \times 12 \mathrm{~mm}$ and were ground and polished, down to a $3 \mu \mathrm{m}$ finish. Notches were introduced into the tensile faces of the beams using a sharp edge in an electrical discharge machine for a few seconds. The result of this process was to produce three or four cracks at the bottom of the notch. An optical micrograph of a typical notch and cracks is shown in Figure 1.

Table 1. Details of the specimens investigated in this work.

\begin{tabular}{cccccccc}
\hline & $\begin{array}{c}\text { Number of } \\
\text { pre-cracks }\end{array}$ & $\begin{array}{c}\text { Maximum pre- } \\
\text { crack length } \\
{[\mu \mathrm{m}]}\end{array}$ & $\begin{array}{c}\text { Loading } \\
\text { temperature } \\
{\left[{ }^{\circ} \mathrm{C}\right]}\end{array}$ & $\begin{array}{c}\text { Peak } \\
\text { maximum } \\
\text { stress [MPa] }\end{array}$ & $\begin{array}{c}\text { Loading } \\
\text { time [s] }\end{array}$ & $\begin{array}{c}K \\
{\left[\mathrm{MPam}{ }^{1 / 2}\right]}\end{array}$ & $\begin{array}{c}\text { Ductility deduced } \\
\text { from [6] }\end{array}$ \\
\hline $\mathbf{0}$ & 4 & 48 & & \multicolumn{5}{c}{ Not loaded } \\
$\mathbf{1}$ & 4 & 51 & 97 & 941 & 1,640 & 11.9 & Semi-brittle \\
$\mathbf{2}$ & 3 & 54 & 185 & 705 & 630 & 9.2 & Ductile \\
$\mathbf{3}$ & 3 & 57 & 212 & 478 & 290 & 6.4 & Ductile \\
$\mathbf{4}$ & 4 & 61 & 212 & 716 & 690 & 9.9 & Ductile \\
$\mathbf{5}$ & 3 & 70 & 212 & 1,072 & 1,380 & 15.9 & Ductile
\end{tabular}

The notched beams were then subjected to four-point bend loading at the same approximately constant strain rate. The load-displacement data were used with an assumed Young's modulus of $400 \mathrm{GPa}$ to deduce the strain rate to be $4( \pm 2) \times 10^{-6} \mathrm{~s}^{-1}$. The rate at which specimens were strained in this current work is the same as the specimens for which Giannattasio and Roberts [6,7] reported a strain rate as $4 \times 10^{-5} \mathrm{~s}^{-1}$. This discrepancy arose because some elements of the compliance of the test 
apparatus were not taken into account when calibrating the strain rate in the previous work [6,7]. The correct strain rates are those given in the current paper. For this strain rate and testing configuration, Giannattasio and Roberts found $T_{B D T}$ to be $120^{\circ} \mathrm{C}$ [6], so the bend tests in this present study were performed, under argon atmosphere, at temperatures above and below this transition temperature. The load on the specimen was increased to a known value below $\sigma_{\text {fracture }}$, at which point the specimen was rapidly unloaded at the testing temperature. After bending, one side of the beam was then ground and polished to a $40 \mathrm{~nm}$ colloidal silica finish.

The details of the specimens produced are given in Table 1, which also includes details of a control specimen which was notched and processed in the same way as all the other specimens, but which was not subjected to an applied stress.

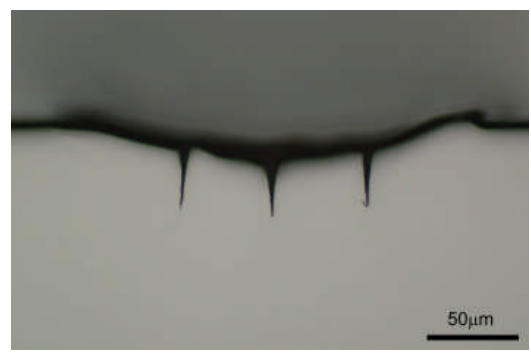

Figure 1. Optical micrograph showing notch and cracks in a typical specimen.

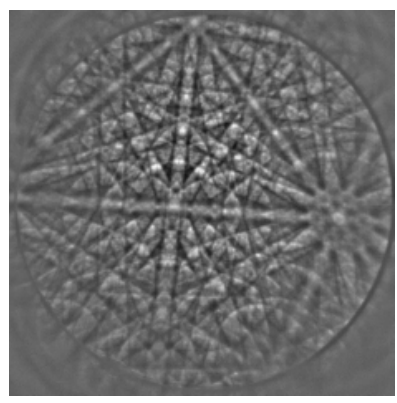

Figure 2. Kikuchi pattern from unstrained region of a typical specimen.

\section{$2.2 E B S D$}

EBSD was performed in a JEOL JSM-6500F scanning electron microscope operating with a $20 \mathrm{kV}$ accelerating voltage and a beam current of approximately $16 \mathrm{nA}$. Specimens were tilted to $70^{\circ}$ and the electrons backscattered from the highly-polished sample formed patterns on a fluorescent screen which were captured by a CCD camera as 1024 x 1024 pixel images. An image of one of these patterns is shown in Figure 2. An array of such patterns was recorded at approximately $1 \mu \mathrm{m}$ intervals in the vicinity of the cracks. The number of patterns recorded depended on the scan area chosen (which in turn depended on the expected extent of deformation), and was typically around 20,000.

The array of patterns was then analysed off-line with CrossCourt software [10], which uses crosscorrelation based methods developed by Wilkinson, Meaden and Dingley [11]. Cross-correlation analysis significantly improves the angular resolution of the EBSD technique. The method allows the elastic strain tensor and lattice rotations to be determined. However, in this application the plastic deformation dominates; elastic strains are small compared to the rotations and so are not shown here. The full three-dimensional lattice rotation is mapped as a function of position in the vicinity of the crack-tip. The system axes are defined as $x_{1}$ along the tensile face of the specimen, $x_{2}$ along the direction of the crack and $x_{3}$ out of the section plane. The rotations in each plane are referred to as $w_{12}$ about the $x_{3}$ axis, (i.e. within the $x_{1}-x_{2}$ plane), $w_{23}$ about the $x_{1}$ axis (within the $x_{2}-x_{3}$ plane) and $w_{31}$ about the $x_{2}$ axis (within the $x_{3}-x_{1}$ plane).

\section{Results}

\subsection{Rotations about three perpendicular axes}

The magnitude of rotations of the crystal about three orthogonal axes in the vicinity of the crack-tip is plotted for Specimen 3 in Figure 3. It can be seen that the magnitude of the in-plane rotations, $w_{12}$, is significantly greater than those out of the sample section plane, i.e. rotations $w_{23}$ and $w_{31}$. This behaviour was seen in all the other specimens investigated. 
The in-plane rotation, $w_{12}$, for Specimen 3 shows rotations of opposite sign on either side of the crack. This is caused by the combined action of the notching process and the opening of the crack during loading. More interestingly, the maps also show in-plane rotations of the crystal emanating from the crack-tip at approximately $45^{\circ}$. These rotations delineate the crack-tip plastic zone and are due to dislocation activity on $\{110\}$ planes. For this sample, the plastic zone around the central (longest) crack extends for approximately $30 \mu \mathrm{m}$ and is associated with in-plane rotations of the crystal of order 0.01 radians.

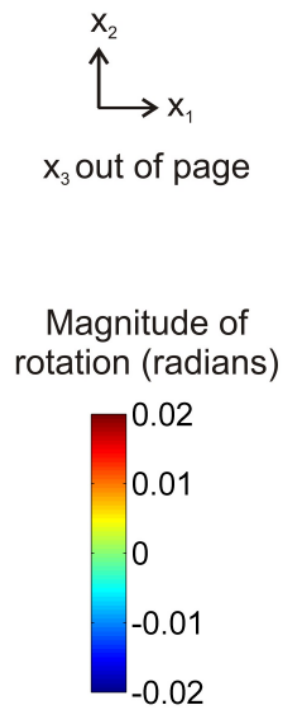

(a) $\mathbf{w}_{12}$ (rotation in $\mathbf{x}_{1}-\mathbf{x}_{2}$ plane)

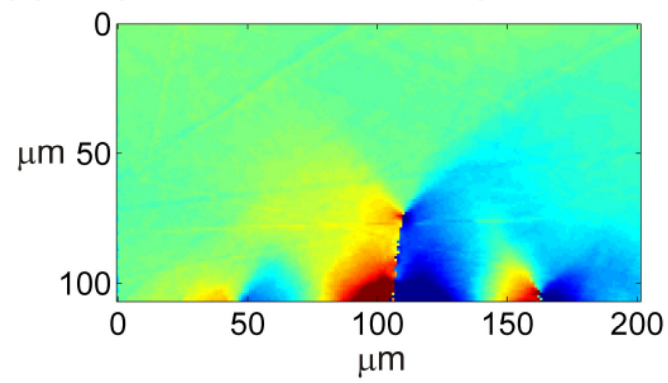

(b) $\mathbf{w}_{23}$ (rotation in $\mathbf{x}_{2}-\mathbf{X}_{3}$ plane)

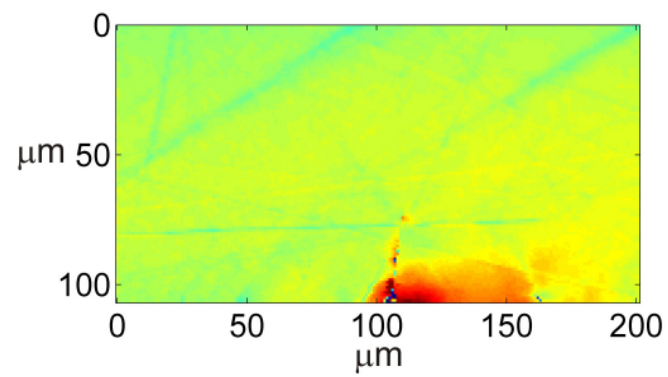

(c) $\mathbf{w}_{31}$ (rotation in $\mathbf{x}_{3}-\mathbf{x}_{1}$ plane)

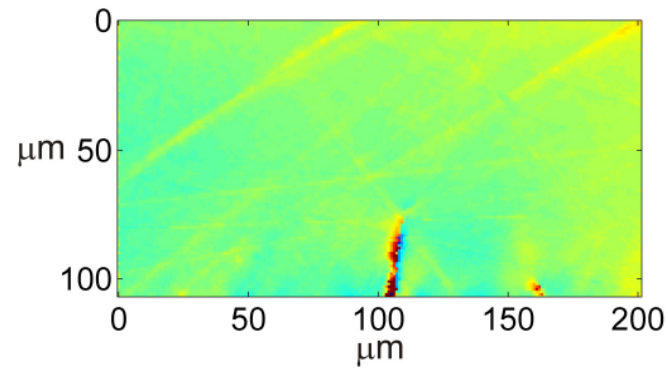

Figure 3. Rotations of the crystal for Specimen 3 about (a) the $\mathrm{x}_{3}$ axis $\left(w_{12}\right)$, (b) the $\mathrm{x}_{1}$ axis $\left(w_{23}\right)$ and $(\mathrm{c})$ the $\mathrm{x}_{2}$ axis $\left(w_{31}\right)$.

\subsection{Effects of loading conditions}

In-plane rotation maps for the samples in Table 1 are shown in Figure 4. For Specimen 0, the control which was not mechanically loaded, the in-plane rotations were confined to the wake of the crack. For all other specimens, plastic zones were found to extend from the crack-tips. Approximate measurements of the sizes of the plastic zones and the in-plane rotations are given in Table 2.

Specimen 1 was deformed at $97^{\circ} \mathrm{C}$; which is below $T_{B D T}$ for the strain rate and testing configuration used. For this specimen, the in-plane rotation map reveals a highly-asymmetric plastic zone extending much further from the crack-tip on side than on the other.

Specimens 2 to 5 were deformed at temperatures above $T_{B D T}$. For all these specimens approximately symmetric plastic zones were found to extend from the crack-tip at $45^{\circ}$ to the crack direction. Specimens 3 to 5 were deformed to different values of $K$ at the same temperature. It can be 
seen that the size of the plastic zone depends upon the value of $K$. The magnitude of the in-plane rotation is also dependent on the applied $K$, with larger rotations observed at for the more highly loaded crack.
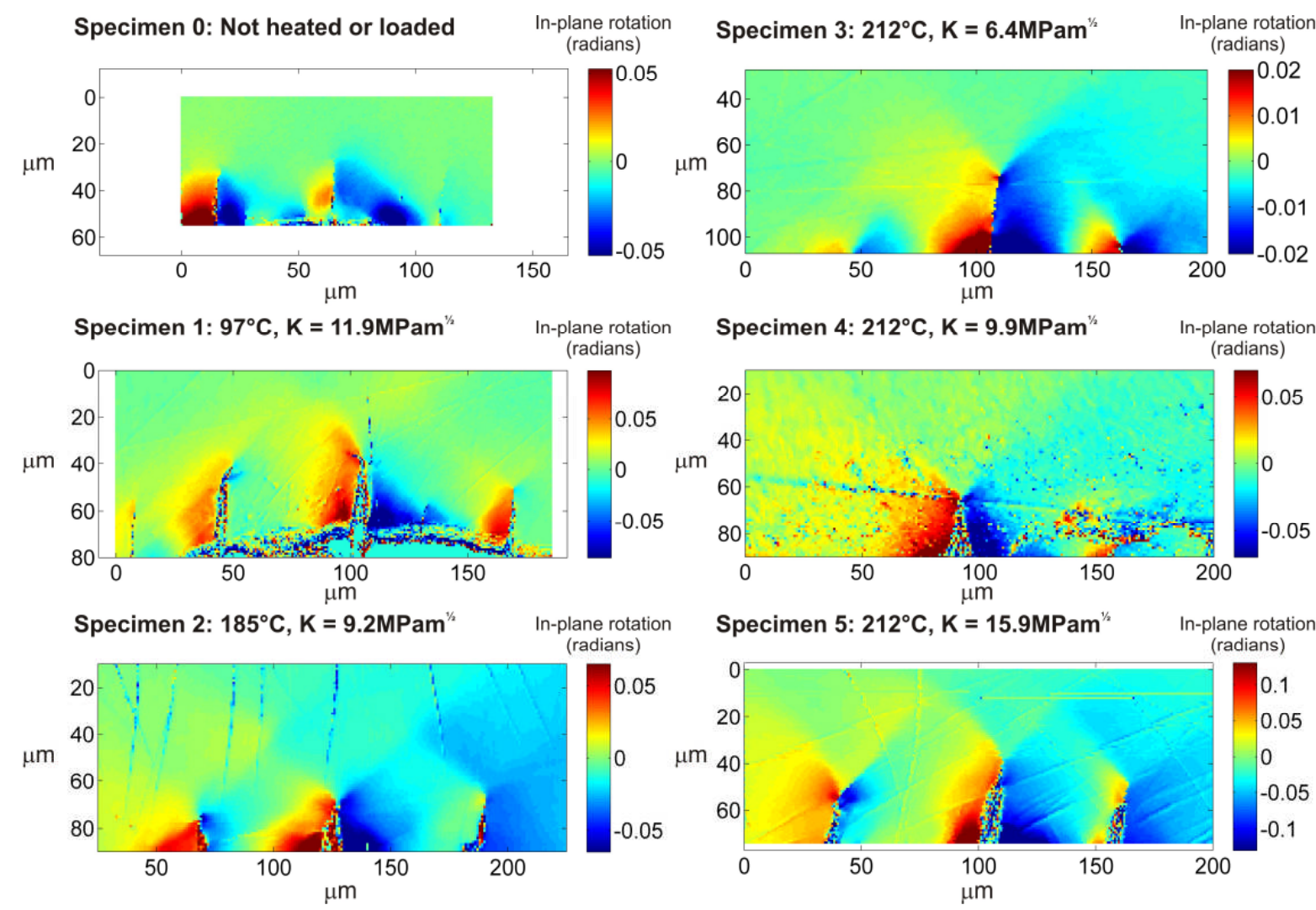

Figure 4. In-plane rotation maps, $w_{12}$, for the samples specified in Table 1.

Table 2. Properties of plastic zones extending from central crack-tips.

\begin{tabular}{|c|c|c|c|c|}
\hline & $\begin{array}{c}\text { Loading } \\
\text { temperature }\left[{ }^{\circ} \mathrm{C}\right]\end{array}$ & $\begin{array}{c}K \\
{\left[\mathrm{MPam}^{1 / 2}\right]}\end{array}$ & $\begin{array}{l}\text { Plastic zone } \\
\text { size }[\mu \mathrm{m}]\end{array}$ & $\begin{array}{c}\text { In-plane rotation } \\
\text { [radians] }\end{array}$ \\
\hline $\mathbf{0}$ & \multicolumn{2}{|c|}{ Not loaded } & 0 & 0 \\
\hline 1 & 97 & 11.9 & $20 \pm 20$ & $\sim 0.05$ \\
\hline 2 & 185 & 9.2 & $20 \pm 20$ & $\sim 0.03$ \\
\hline 3 & 212 & 6.4 & $30 \pm 20$ & $\sim 0.01$ \\
\hline 4 & 212 & 9.9 & $40 \pm 30$ & $\sim 0.03$ \\
\hline 5 & 212 & 15.9 & $>50$ & $\sim 0.05$ \\
\hline
\end{tabular}

\section{Discussion}

The results presented in Figures 3 and 4 show that cross-correlation of EBSD patterns provides an effective way of studying plastic zones around loaded crack-tips in single-crystal metals. Figure 3 shows that in-plane crystal rotations, $w_{12}$, dominate over other rotations. This is to be expected given that observations are made on a surface prepared by grinding in from the side face of the sample to sufficient depth for a state of plane strain to predominate. Although the Burgers vectors of the highly stressed slip systems all have components out of the section plane [9], the contributions of dislocations 
with opposite out-of-pane contributions are expected to be matched so that the net out-of-plane component is near-zero.

From the in-plane rotation maps, it is possible to determine the approximate size of the plastic zones and the magnitudes of the in-plane rotation. The results presented in Figure 4 show that, for specimens deformed above $T_{B D T}$, a plastic zone extends from the crack-tip along $\{110\}$ planes. For specimens deformed at the same temperature, $212^{\circ} \mathrm{C}$, the size of the plastic zone and the rotation of the crystal therein depends upon $K$. For a given temperature (and hence friction stress) the size of the plastic zone should increase as approximately the square of the applied stress intensity factor [9]. Although an increase in plastic zone size is observed, its rate of increase does not seem as strong as expected. Reasons for this may include elastic interactions between the multiple cracks within each notch, interactions between plastic zones generated by the adjacent cracks, and the fact that the cracks are short compared to the size of the plastic zones generated.

It should be noted that the EBSD results presented here imply an unexpected property of the dislocations in the plastic zones. It can be seen that from the in-plane rotation maps, $w_{12}$, in the Figures 3 and 4, the magnitude of the in-plane rotation changes abruptly perpendicular to the arm of the plastic zone along $\{110\}$ planes. To give rise to such a rotation it would be necessary for the Burgers vectors of the dislocations to be normal to the direction of dislocation motion away from the crack-tip. The physical mechanism behind this is not clear. The work continues with a transmission electron microscopy investigation of the dislocation-containing region of the samples.

\section{Conclusions}

EBSD has been used to study the plastic-zones around pre-loaded crack-tips in pure single-crystal tungsten. In-plane rotations of the lattice have been found to dominate over out of plane rotations. For specimens deformed above $T_{B D T}$, in-plane rotation maps reveal clear plastic zones along $\{110\}$ planes. The size of the plastic zones depends upon temperature and $K$. The results presented here form the basis of a preliminary study and the mechanisms of the dislocation motion away from the crack-tip are the subject of further investigation.

\section{Acknowledgments}

The authors are grateful to the European Union FP6 Integrated Project 'Extremat' (contract NMP-CT2004-500253) and to UKAEA Culham for project funding. Additionally, JDM is the grateful recipient of a Royal Academy of Engineering/ EPSRC Research Fellowship and a Junior Research Fellowship at St Anne's College, Oxford.

\section{References}

[1] H. Bolt, V. Barabash, W. Krauss, J. Linke, R. Neu, S. Suzuki, N. Yoshida 2004 Journal of Nuclear Materials 329-333 66

[2] P. Gumbsch, J. Riedle, A. Hartmaier, H.F. Fischmeister 1998 Science 2821293

[3] H.W. Schadler 1964 Acta Metall. 12861

[4] A.S. Argon, S. R. Maloof 1966 Acta Metall. 141449

[5] D. Brunner 2000 Mater. Trans. JIM 41152

[6] A. Giannattasio, S.G. Roberts 2007 Philos. Mag. 872589

[7] A. Giannattasio, S.G. Roberts unpublished

[8] P.B. Hirsch, S.G. Roberts 1991 Philos. Mag. 6455.

[9] E. Tarleton, S.G. Roberts Philos. Mag. in press

[10] CrossCourt code written by BLG Productions, www.blgproductions.co.uk

[11] A.J. Wilkinson, G. Meaden, D.J. Dingley 2006 Materials Science and Technology 221271 